\title{
Concurrent coronary artery and subclavian arterial aneurysms in Takayasu arteritis
}

\author{
Anoop Ayyappan ${ }^{1 *} \mathbb{D}$, Arun Gopalakrishnan ${ }^{2}$ and Shivanesan Pitchai ${ }^{3}$
}

\begin{abstract} Takayasu arteritis. Takayasu arteritis patient. aneurysm, Computed tomography, Magnetic resonance imaging

\section{Background}

Takayasu arteritis is a large vessel vasculitis involving the aorta and its branches with occasional coronary artery involvement. Clinical presentation as multiple aneurysms and distal embolic complication from aneurysm usually makes the possibility of arteritis less likely. We present a case who presented with complications secondary to coronary occlusion with aneurysms in coronary arteries in a male patient, who was subsequently diagnosed with Takayasu arteritis.
\end{abstract}

Background: Aneurysmal coronary artery involvement and subclavian artery aneurysm are extremely uncommon in

Case presentation: We present a case with concurrent coronary artery and subclavian artery aneurysms.

Conclusions: This case report stresses multimodality and multisystem imaging in Takayasu arteritis to know the disease load in the patient and to know the possibility of a rare type of involvement (medium and large vessel) in

Keywords: Takayasu arteritis, Left main coronary artery aneurysm, Coronary artery aneurysm, Subclavian artery

\section{Case presentation}

A 28 years old man with no conventional risk factors for coronary artery disease presented with history of NYHA functional class II exertional dyspnea for 2 months. He did not have angina or documented acute coronary syndromes in the past. On detailed interrogation, easy fatigability of both upper limbs with mild claudication was noted. The arterial pulses were

\footnotetext{
*Correspondence: anoop123a@gmail.com

1 Department of Imaging Sciences and Interventional Radiology, Sree Chitra Tirunal Institute for Medical Sciences and Technology, Trivandrum, Kerala 695011, India

Full list of author information is available at the end of the article
}

absent in both upper limbs suggesting a pulseless stage of disease. The blood pressure in the upper limbs was $80 / 60 \mathrm{~mm} \mathrm{Hg}$ against a pressure of $120 / 80 \mathrm{~mm} \mathrm{Hg}$ in the right lower limb suggesting normotensive status. Bilateral subclavian bruit and carotid artery tenderness were noted. Electrocardiography showed loss of $\mathrm{R}$ waves in anterior precordial leads and $\mathrm{T}$ wave changes suggestive of remote anterior wall myocardial infarction. The anterior and basal anteroseptal segments were hypokinetic with preserved thickness in echocardiogram. Arterial doppler interrogation showed bilateral subclavian artery stenosis with dampened distal flow. The abdominal aorta was also aneurysmal with stenotic involvement of the celiac axis, superior mesenteric artery and right renal artery. A CT peripheral angiogram and MR angiogram confirmed short segment occlusion in bilateral subclavian arteries and saccular aneurysm involving proximal left subclavian $\operatorname{artery}$ (Fig. 1), left main coronary artery (LMCA) aneurysm (Fig. 2), aneurismal dilation of right coronary ostium (Figs. 1, 2)and abdominal aortic aneurysm (Fig. 3). There was a fusiform aneurysm involving LMCA with total occlusion of left anterior descending artery. The proximal right coronary artery (RCA) also showed a small aneurysm followed by significant 


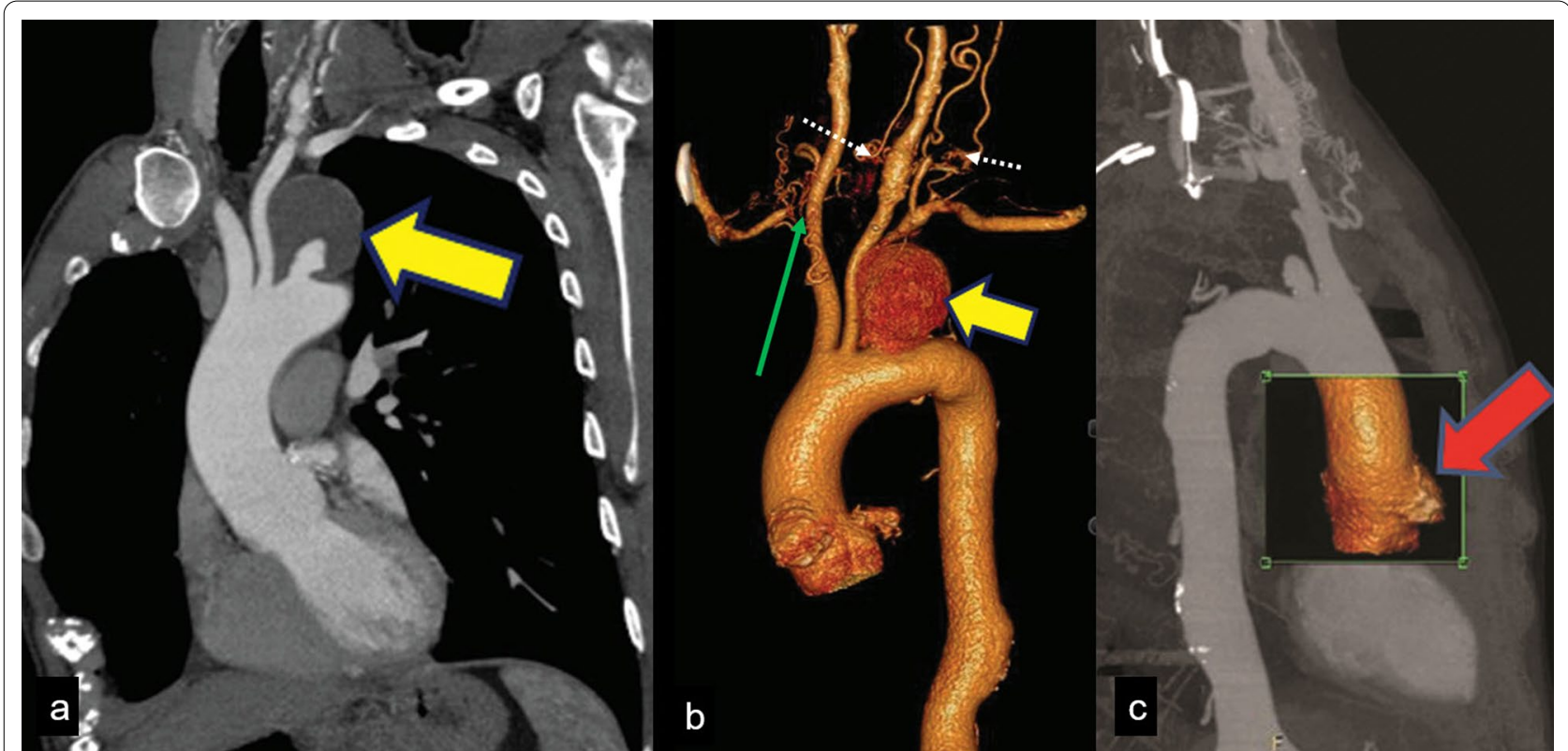

Fig. 1 a CT angiogram in coronal oblique plane showing aneurysm (yellow solid arrow) in proximal left subclavian artery with thrombosis of lumen. $\mathbf{b}$ Distal to thrombosed aneurysm (yellow solid arrow) left subclavian artery is filled through collaterals from left external carotid artery (white dotted arrow) in VRT image. Note is also made of proximal short segment occlusion of right subclavian artery (green arrow). c CT angiogram VRT image showing aneurysmal dilation of right coronary ostium (red arrow)

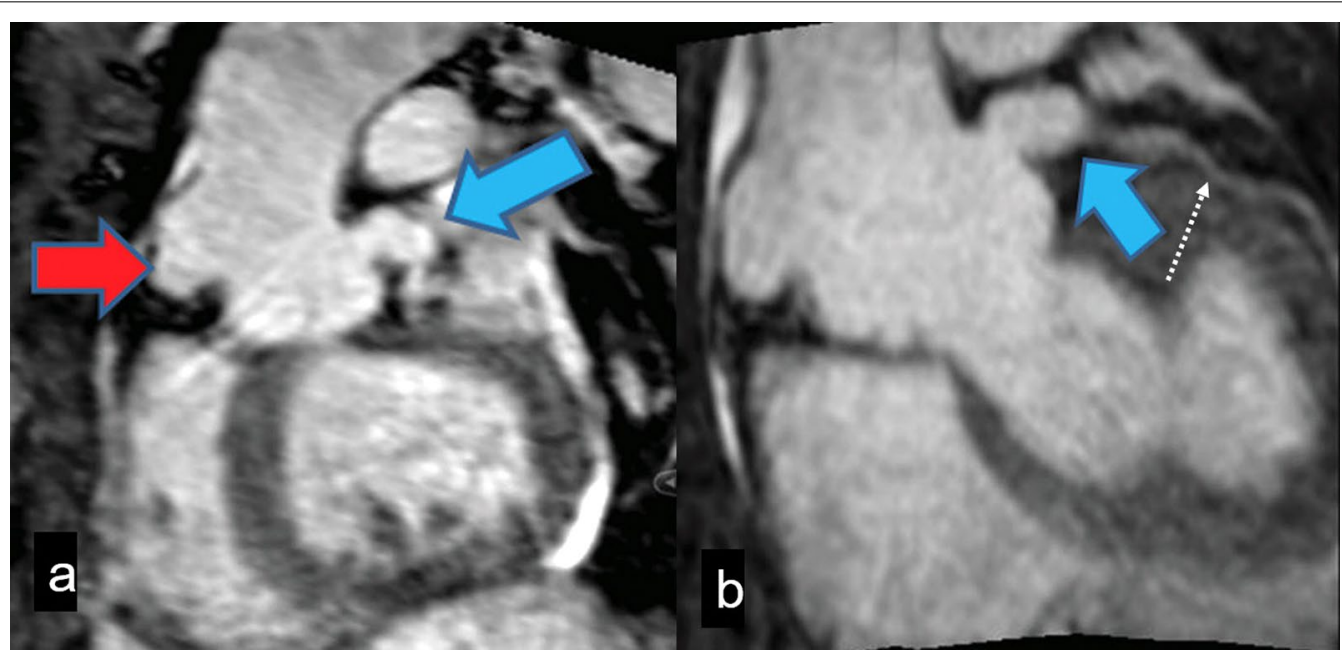

Fig. 2 a Cardiac triggered navigator-gated 3D bSSFP Noncontrast MR angiogram MIP image in parasagittal view showing aneurysmal dilation of right coronary ostium (red arrow) and fusiform aneurysmal dilation of Left main coronary artery (blue arrow). b MR angiogram in oblique coronal plane showing fusiform aneurysmal dilation of Left main coronary artery (blue arrow) followed by short segment total occlusion of proximal left anterior descending artery and diffusely narrow calibre distal vessel (dotted arrow)

stenosis distally. Work up for tuberculosis was negative. Both C-reactive protein $(20 \mathrm{mg} / \mathrm{dl})$ and erythrocyte sedimentation rate $(84 \mathrm{~mm} / \mathrm{h})$ were elevated. A diagnosis of Takayasu arteritis type 5 (Fig. 3) was made based on American College of Rheumatology criteria [1]. He was started on lifelong low dose Aspirin (75 mg once daily) to prevent thrombosis and embolic complications and heart failure medications. He was also started on disease modifying drugs for controlling disease activity of Takayasu arteritis with regular follow-ups. Considering poor results for surgical and interventional management in Takayasu arteritis, surgical interventions 


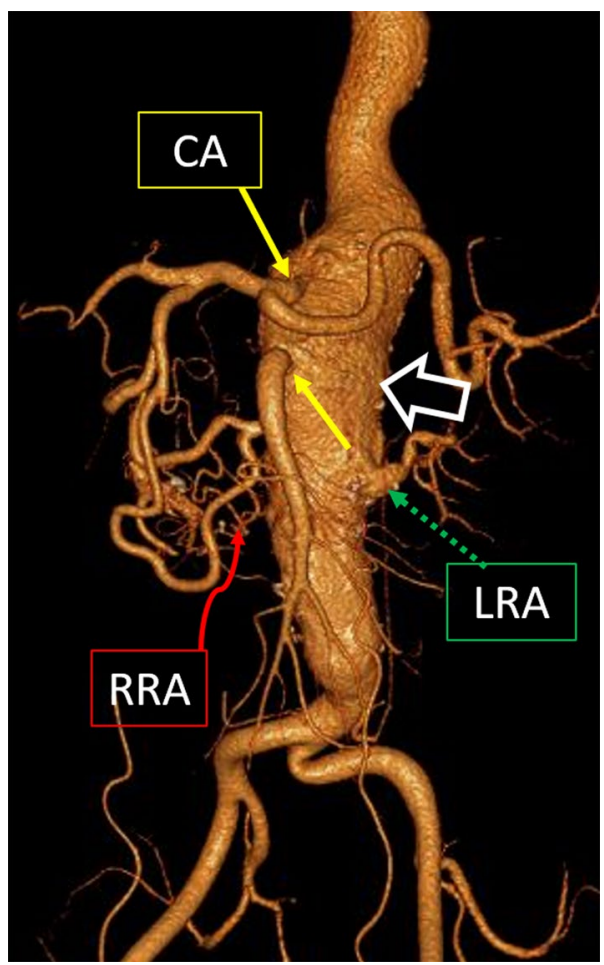

Fig. 3 Volume rendered CT angiogram in frontal projection showing aneurysmal dilation of abdominal aorta (white open arrow) and mild stenoses at ostia of celiac artery (CA) and superior mesenteric arteries (yellow arrows). This patient had atrophy of right kidney secondary to right renal artery (RRA) occlusion (red curved arrow) with single functional kidney on left side supplied by left renal artery (LRA) green dotted arrow. Tortous iliac arteries were noted to have normal diameters

were deferred till he becomes more symptomatic (myocardial infarction or limb claudication) or lesion progre sses.

\section{Discussion}

Coronary artery involvement in Takayasu arteritis has been documented in up to $10 \%$ of cases [2]. This may occur as coronary stenosis, occlusion, diffuse or focal coronary arteritis or aneurysm formation [3]. Coronary artery aneurysm formation is distinctly uncommon in aortoarteritis, with fewer than $1.5 \%$ of Takayasu arteritis cases associated with it [4].Multiple mechanisms have been postulated for aneurysm formation in this scenario, one being accelerated atherosclerosis (following Systemic hypertension and systemic inflammatory response occurring in Takayasu Arteritis) and the other due to Arteritis proper $[5,6]$. The chronic inflammation involving large to medium sized arteries and related edema have also been documented to result in active vasculitis leading to coronary and vascular lesions [5]. It is believed that the majority of coronary lesions in aortoarteritis are related to the extension of chronic aortic inflammation into the coronary media and adventitia, as substantiated by predominant ostial coronary involvement. [5] It is known that coronary artery aneurysms are associated with increased mortality [6]. While in general, coronary artery aneurysms have been most commonly noted in the $\mathrm{RCA}$, both the left and right have been equally implicated in coronary aneurysms in Takayasu arteritis (Fig. 2) [7, 8].

Other diseases which may present with peripheral arterial aneurysm and in the setting of coronary artery aneurysm are Kawasaki disease, atherosclerosis and polyarteritis nodosa. However, characteristic involvement of the aorta and its branches in our case clinched the diagnosis of Takayasu arteritis.

While aneurysmal involvement of the aorta is common in aortoarteritis, subclavian artery aneurysm is extremely rare [9]. The subclavian arteries generally show stenotic involvement of the proximal or middle third in aortoarteritis. In the index case, there was aneurysmal involvement of the ostioproximal left subclavian artery with short segment occlusion distal to it. Distally the subclavian artery assumed normal caliber with contribution from cervical collaterals Fig. 1c.

Identification of disease activity is also important in these situations for which MR contrast administration and T2 weighted imaging are useful. [10]. Interventions are deferred in the active phase of the disease.

\section{Conclusions}

This case constitutes a rare report of simultaneous aneurysmal involvement of the subclavian artery and coronary artery in Takayasu arteritis though it's considered as a large vessel vasculitis. Thorough clinical examination and screen for multivessel involvement by choice of appropriate imaging modality is essential in management.

\section{Abbreviations}

3D bSSFP: 3-Dimensional balanced steady state free precession; CT: Computed tomography; LMCA: Left main coronary artery; MIP: Maximum Intensity Projection; MR: Magnetic resonance; NYHA: New York Heart Association; RCA: Right coronary artery; VRT: Volume rendered technique.

\section{Acknowledgements}

Nil.

\section{Authors' contributions}

Author AA, AG contributed in concept, drafting article, critical revision of article and approval of article, whereas SP contributed in drafting article, critical revision of article and approval of article. All authors read and approved the final manuscript.

\section{Funding}

No funding/ financial benefits received.

Availability of data and materials Not applicable. 


\section{Declarations}

Ethics approval and consent to participate

Institutional Ethical Approval was obtained. Consent to participate is considered as consent to publish as ours is a case report.

\section{Consent to publish}

Appropriate consents for publishing the clinical and imaging details of the patient were obtained.

\section{Competing interests}

The authors declare that they have no competing interests.

\section{Author details}

'Department of Imaging Sciences and Interventional Radiology, Sree Chitra Tirunal Institute for Medical Sciences and Technology, Trivandrum, Kerala 695011, India. ${ }^{2}$ Department of Cardiology, Sree Chitra Tirunal Institute for Medical Sciences and Technology, Trivandrum, Kerala 695011, India. ${ }^{3}$ Division of Vascular Surgery, Sree Chitra Tirunal Institute for Medical Sciences and Technology, Trivandrum, Kerala 695011, India.

Received: 14 August 2021 Accepted: 19 September 2021 Published online: 27 September 2021

\section{References}

1. Arend WP, Michel BA, Bloch DA, Hunder GG, Calabrese LH, Edworthy SM, Fauci AS, Leavitt RY, Lie JT, Lightfoot RW, Masi AT, McShane DJ, Mills JA, Stevens MB, Wallace SL, Zvaifler NJ (2010) The American College of Rheumatology 1990 criteria for the classification of takayasu arteritis. Arthritis Rheum 33:1129-1134. https://doi.org/10.1002/art.1780330811
2. Lupi-Herrera E, Sánchez-Torres G, Marcushamer J, Mispireta J, Horwitz S, Vela JE (1977) Takayasu's arteritis. Clinical study of 107 cases. Am Heart J 93:94-103

3. Endo M, Tomizawa Y, Nishida H, Aomi S, Nakazawa M, Tsurumi Y, Kawana M, Kasanuki H (2003) Angiographic findings and surgical treatments of coronary artery involvement in Takayasu arteritis. J Thorac Cardiovasc Surg 125:570-577. https://doi.org/10.1067/mtc.2003.39

4. Hartnell GG, Parnell BM, Pridie RB (1985) Coronary artery ectasia. Its prevalence and clinical significance in 4993 patients. Br Heart J 54:392-395

5. Matsubara O, Kuwata T, Nemoto T, Kasuga T, Numano F (1992) Coronary artery lesions in Takayasu arteritis: pathological considerations. Heart Vessels Suppl 7:26-31

6. Baman TS, Cole JH, Devireddy CM, Sperling LS (2004) Risk factors and outcomes in patients with coronary artery aneurysms. Am J Cardiol 93:1549-1551. https://doi.org/10.1016/j.amjcard.2004.03.011

7. Syed M, Lesch M (1997) Coronary artery aneurysm: a review. Prog Cardiovasc Dis 40:77-84. https://doi.org/10.1016/S0033-0620(97)80024-2

8. Topaz O, DiSciascio G, Cowley MJ, Goudreau E, Soffer A, Nath A, Lanter P, Vetrovec GW (1991) Angiographic features of left main coronary artery aneurysms. Am J Cardiol 67:1139-1142. https://doi.org/10.1016/00029149(91)90881-K

9. Chiou A (1999) Subclavian artery aneurysm: an unusual manifestation of Takayasu's arteritis. Cardiovasc Surg 7:310-314. https://doi.org/10.1016/ S0967-2109(98)00171-9

10. John RA, Keshava SN, Danda D (2017) Correlating MRI with clinical evaluation in the assessment of disease activity of Takayasu's arteritis. Int J Rheum Dis 20:882-886. https://doi.org/10.1111/1756-185X.12967

\section{Publisher's Note}

Springer Nature remains neutral with regard to jurisdictional claims in published maps and institutional affiliations.

\section{Submit your manuscript to a SpringerOpen ${ }^{\circ}$ journal and benefit from:}

- Convenient online submission

- Rigorous peer review

- Open access: articles freely available online

- High visibility within the field

- Retaining the copyright to your article

Submit your next manuscript at $\boldsymbol{\nabla}$ springeropen.com 